\title{
Las sucesiones terrígenas del Triásico superior y la base del Jurásico del Alto Muluya (Marruecos): estratigrafía y contexto geodinámico
}

\author{
Terrigenous successions of the late Triassic-early Jurassic in the High \\ Moulouya (Morocco): stratigraphy and geodynamic context
}

\author{
Z. Saâdi ${ }^{1}$, B. Fedan², M. Laadila ${ }^{3}$, O. Azzouz ${ }^{4}$, C. Sanz de Galdeano ${ }^{5}$
}

\begin{abstract}
RESUMEN
Las cuencas triásico-jurásicas del Alto Muluya han registrado la evolución del proceso de "rifting" que ocurrió en el Atlas: una apertura en la dirección SO-NE, una sedimentación continental sintectónica y un volcanismo activo. En este proceso se formaron cuencas a modo de sucesivos escalones asimétricos, que fueron hundiéndose a lo largo del tiempo. Estas cuencas, en especial durante la formación de las series terrígenas (argilites salifères supérieures en la literatura francesa de la región: Michard, 1976; Kaoukaya, 1987) de la base del Jurásico, muestran una organización típica de relleno continental sintectónico, con intercalaciones volcanosedimentarias y volcanoclásticas, generalmente con sedimentos gruesos hacia la base, progresivamente más finos hacia el techo. El origen de estos depósitos se debe a la erosión de relieves próximos en vías de reactivación. Los depósitos más gruesos, nutridos por cantos volcánicos, forman secuencias de depósitos aluviales y fluviales entre las que se intercalan de forma irregular facies arcillosas de llanura de inundación. El relleno se producía por fuertes descargas intermitentes con una gran cantidad de sedimentos, propias de corrientes torrenciales que depositaban los sedimentos a lo largo de los escarpes originados por fallas. El techo de las series terrígenas muestra un predominio de detríticos finos y no presenta restos volcanoclásticos, lo que refleja el arrasamiento de los relieves en el área fuente y la instauración en el área de depósito de una llanura de inundación. Esto atestigua una homogenización generalizada durante un periodo de calma tectónica. Durante el depósito de las series terrígenas, las rocas piroclásticas se formaron en los sectores tectónicamente activos (Tizi n'Rechou, Tizi n'Toumelba, Tawrirt, Boutkhoubaye et Boumia), donde son potentes y variadas. Este volcanismo tardío se produce en relación con las últimas fases del "rifting" manifestado por el rejuego de estructuras mayores hercinianas y tardi-hercinianas. Así, durante el Triásico y la base del Jurásico la región estuvo sometida a la extensión producida en los estadios iniciales de la apertura del Atlántico central y del Tethys occidental.
\end{abstract}

Palabras clave: Marruecos, región de Boumia-Tizi n’Rechou, Triásico superior-Jurásico basal, dinámica sedimentaria, geodinámica.

\section{ABSTRACT}

The Triassic-Jurassic basins of the High Moulouya area record the evolution of the Atlasic rifting in the SW-NE direction, showing a syntectonic continental sedimentation and an active volcanism. Such mobility has led to the differentiation of asymmetric half basins, whose gradual subsidence took place in successive stages. These basins, particularly during the deposition of the terrigenous successions of the

\footnotetext{
1 École Nationale d'Agriculture de Meknès (ENAM), B.P. S/40, Meknès, Maroc. e-mail: szouhra@yahoo.fr, szohra2008@Hotmail.fr

2 Université Mohammed V-Agdal, Institut Scientifique, Département de Géologie, B.P. 703 Agdal, Rabat, Maroc. Email: fedan@israbat.ac.ma, bouazafedan@yahoo.fr

3 Université Mohammed V-Agdal, Faculté des Sciences, Département des Sciences de la Terre, B.P. 1014 R.P., Rabat, Maroc. Email: laadila@fsr.ac.ma

4 Université Mohammed Premier, Faculté des Sciences, Département de Géologie, B.P. 524 Oujda, Maroc. Email: azzouzomar@yahoo.fr

5 Instituto Andaluz de Ciencias de la Tierra (CSIC-Univ. Granada). Facultad de Ciencias. 18071 Granada España). Email: csanz@ugr.es
} 


\begin{abstract}
base of the Jurassic (early Liassic age), show a typical syntectonic continental infill. It consists of a mixed sedimentation, detrital and volcanoclastic, generally coarse-grained towards the base. Coarser deposits, fed by volcanic clasts, are structured as sequences of spreading river cones, alternating with irregular clay facies of flood plain. They reflect a rejuvenation of the relief, following a reactivation of the faults. The sedimentary infill was provided by intermittent flows with considerable discharge, forming deposits along fault escarpments. The top of the terrigenous series shows a dominance of thin detritic sediments and a lack of volcanoclastics, reflecting the peneplanation of the relief and the creation of a flood plain. It records the widespread homogenization of the prior relief, causing the disappearance of the pre-existing structures during a period of tectonic quiescence. The volcanoclastics, witnessing an explosive volcanism and a concomitant sedimentation, are clustered in the tectonically active regions, where they are thicker and diversified. This late volcanism occurred during the final phases of rifting, owing to the replay of major Hercynian and tardi-hercynian faults. The Triassic-Jurassic period is thus marked by an extensional dynamics controlled by the influence of the first stages of the opening of the central Atlantic and the western Tethys.
\end{abstract}

Key words: Morocco, Boumia-Tizi n'Rechou, latest Trias-earliest Jurassic, sedimentary dynamics, Geodynamics.

\section{Introducción}

En las proximidades del paleoumbral paleozoico del Alto Muluya (región situada entre el Atlas Medio y el Alto Atlas Central) (Fig. 1), las cuencas triásico-jurásicas fueron en gran medida creadas por la tectónica de rifting que afectó a la región del Atlas. Se produjeron entonces importantes cambios paleogeográficos en la región, con formación de nuevos relieves, a la vez que en las cuencas había notables variaciones laterales en su relleno y en sus facies. Sin embargo, a techo de ese relleno, las series terrígenas de la base del Jurásico (en adelante series terrígenas liásicas: STL) - las "argilites salifères supérieures" en la literatura francesa - se caracterizan por mostrar una cierta homogenización litológica y por estar presentes en el conjunto de las cuencas. Aun así, estas STL, depositadas sobre depósitos volcánicos piroclásticos (Chalot-Prat et al., 1986; Ouarhache, 1993, 2002; Ouarhache et al., 1985, 2000, 2002, 2004; Saâdi \& Fedan, 1997b, 1999b; Saâdi et al., 2004a y b, 2006), presentan facies variadas, además de espesores importantes.

El estudio de las STL constituye el objetivo del presente artículo. Se muestran sus variaciones de facies y, particularmente, el control que sobre el relleno tuvo la tectónica que simultáneamente tenía lugar.

\section{Situación geológica}

En la región de Boumia-Tizi n'Rechou, las STL se desarrollan bien. Esta región se sitúa (Fig. 1) al SE de la falla de Aït Oufella, en el borde sur del Atlas Medio Meridional. Allí, sobre un basamento paleo- zoico granitizado, afloran, de más antiguo a más moderno, arcosas, areniscas violáceas y lutitas salíferas inferiores, rocas basálticas, en general doleritas, y las STL (Michard, 1976; Ganzeev et al., 1978; El Wartiti, 1981; Salvan, 1984; Et-Touhami, 1996; Oujidi, 1996; Oujidi et al., 2000). Este conjunto de sedimentos es de una edad que abarca del Triásico a la base del Jurásico (antiguo Líasico, o Liás inferior: Hettangiense-Sinemuriense); en particular, las STL ya corresponden al Lías inferior (Manspeizer et al., 1978; Baudelot \& Charriere, 1983; Courtinat \& Algouti, 1985; Rakus, 1986; Sabaoui, 1987; Baudelot et al., 1990; Sebai et al., 1991; Fiechtner et al., 1992; Lachkar et al., 2000; Bouatmani et al., 2004). Los carbonatos jurásicos situados a techo forman grandes planicies hacia el NO. Estos depósitos están afectados por fallas N-S que cambian de dirección hacia Tizi n'Rechou, donde se paralelizan con la falla de Ait Oufella (FAO) tomando una dirección SO-NE (Figs. 1 y 2), con buzamiento al NO (Saâdi, 1996). La mayor parte de estas fallas submeridianas a SONE que compartimentan el basamento paleozoico han controlado la formación de las cuencas triásicojurásicas.

\section{Descripción de las formaciones e interpretación sedimentaria}

Las STL estudiadas en la región de Boumia-Tizi n'Rechou reposan sobre la superficie irregular del techo del complejo basáltico de edad comprendida entre el Triásico superior y la base del Jurásico (Manspeizer et al., 1978; Sebai et al., 1991; Fiechtner et al., 1992; Marzoli et al., 2004; Verati et al., 2005). En esa superficie irregular existen grietas 


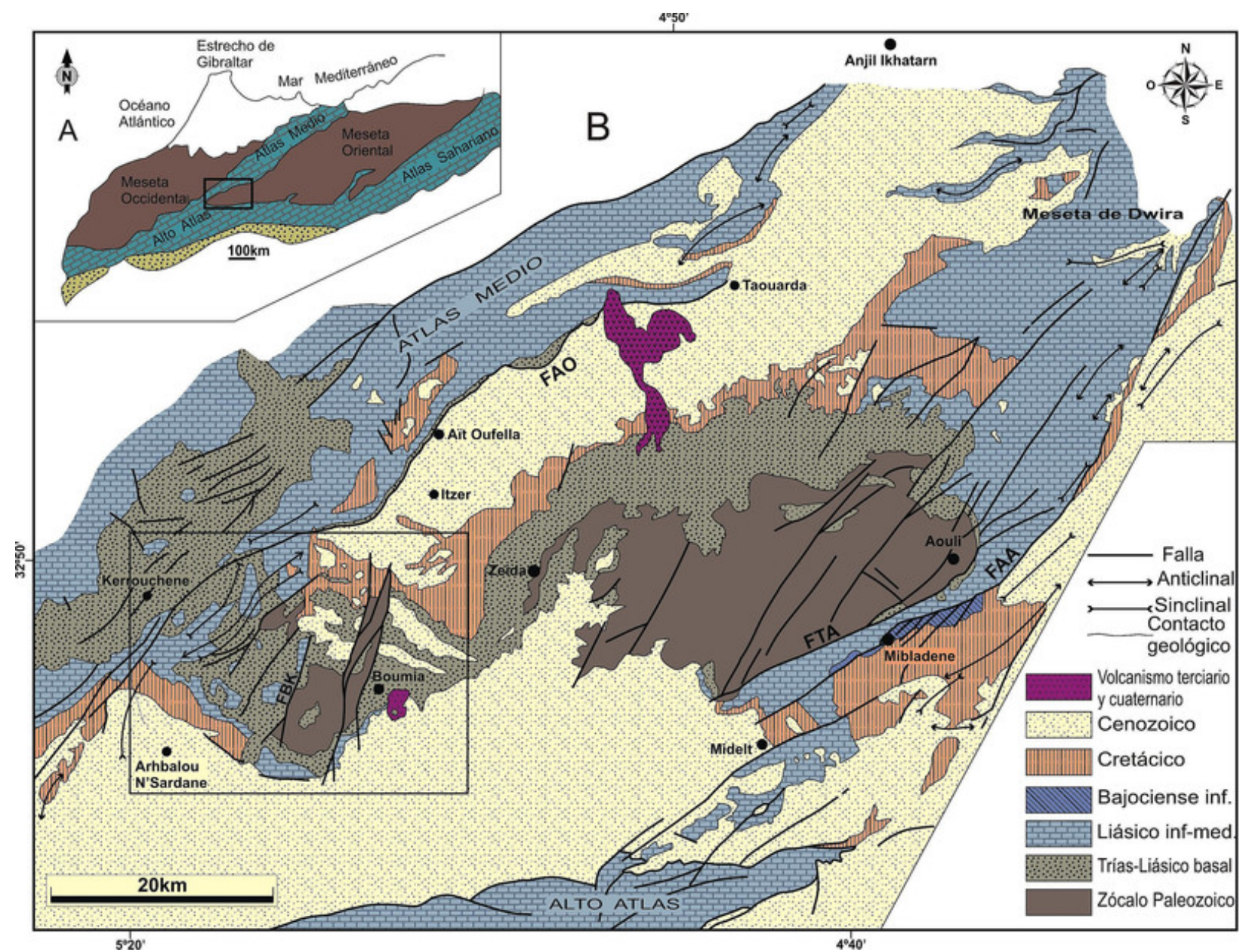

Fig. 1.-El Alto Muluya y el borde meridional del Atlas Medio. A- Mapa de localización. B- Esquema geológico simplificado de la región en la que se sitúa el área estudiada. FAO: falla de Aït Oufella, FBK: falla de Boutkhoubaye, FTA: falla de Taddammout-Aouli, FAA: flexure/falla de Amghouzif. El recuadro indica la posición de la figura 2.

rellenas por lutitas rojas. Las STL se desarrollan muy bien al NO (105 $\mathrm{m}$ de potencia en Tizi n'Rechou: columna TN) mientras que al SE (Boutkhoubaye y Boumia) tienen espesores reducidos (Fig. 3). En ellos se distinguen tres unidades litoestratigráficas (equivalentes a las de Ouarhache, 2002) que, de abajo arriba, son las formaciones de Tizi n'Rechou, Tizi n'Toumelba y Aghbalou Oumlil (Fig. 3).

La formación inferior (Fm. Tizi n'Rechou) es la que ocupa mayor extensión regional, y muestra espesores y facies lateralmente variables, que testimonian una estructuración compleja de la cuenca: facies aluviales, más proximales al SE, que evolucionan a facies más distales, localmente ausentes hacia el NO (Tizi n'Toumelba: corte Tt2). La formación de Tizi n'Toumelba, bien desarrollada en el centro y NO del área de estudio (Tawrirt, Tizi n'Rechou y Tizi n'Toumelba: respectivamente TW, TN y Tt1 en la Fig. 3), se encuentra muy reducida en Boutkhoubaye (BK en Fig. 3) y está ausente en Boumia y, localmente también, en Tizi n'Toumelba (BM y Tt2 en la Fig. 3). La formación superior, de Aghbalou Oumlil, cubre toda la región. Los carbonatos de la formación de Lissit del Jurásico inferior (Sinemuriense superior) cubren esa formación y atestiguan una inundación marina de la región (Figs. 3, 5 y 6).

La formación de Tizi n'Rechou está principalmente constituida (Fig. 4) por conglomerados, arenas y lutitas de colores predominantemente rojos. Estos depósitos tienen un origen aluvial s.l. y pueden contener intercalaciones de rocas piroclásticas y 


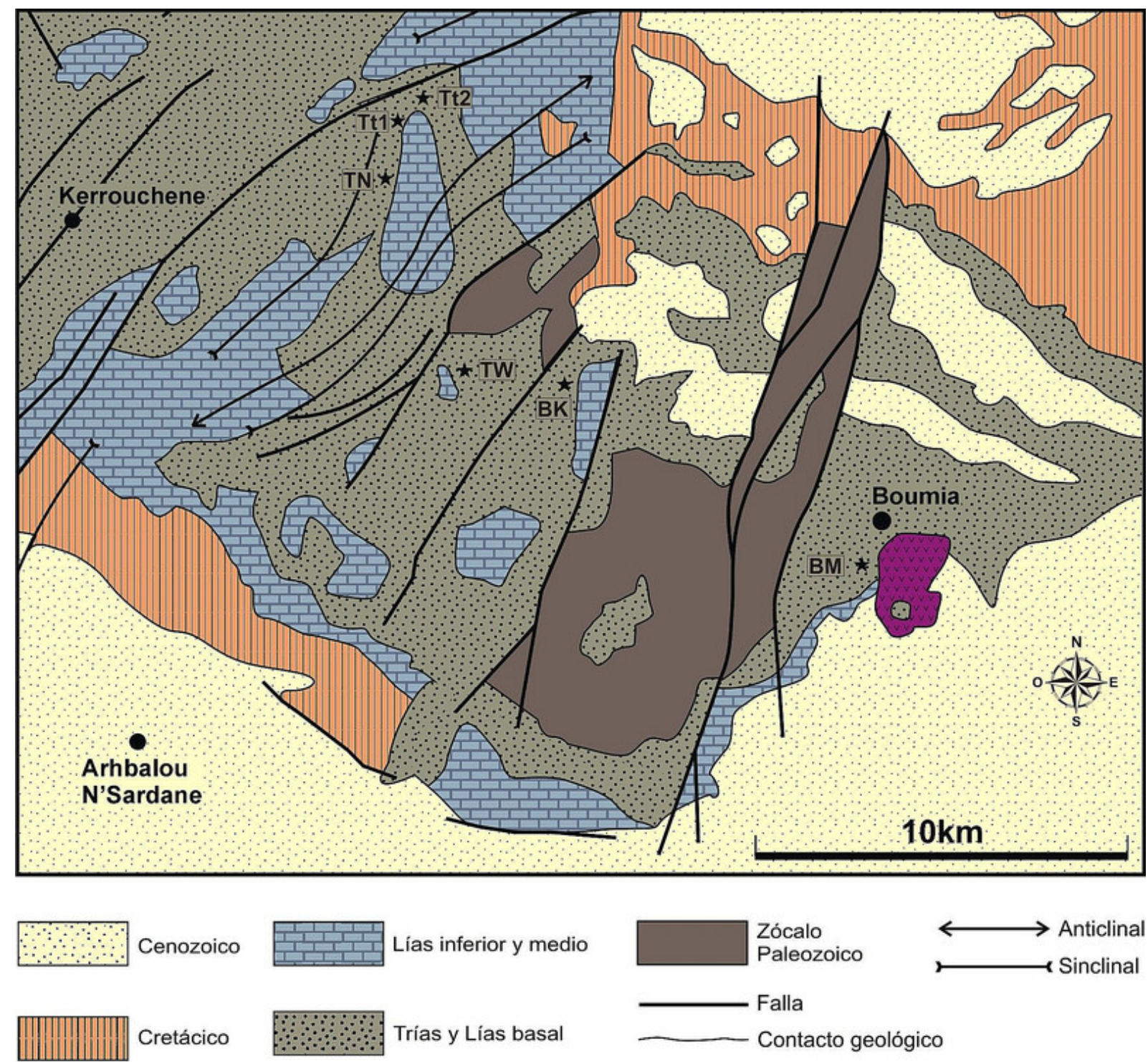

Fig. 2.-Detalle de la figura $1 \mathrm{~B}$, donde se ha representado la zona de estudio con la localización de las columnas de la Fig. 3 y del corte de la Fig. 6. TN: Tizi n’Rechou, Tt: Tizi n'Toumelba, TW: Tawrirt, BK: Boutkhoubaye, BM: Boumia. La línea de dirección NO-SE indica la posición aproximada del esquema de la figura 6.

algunos niveles delgados de carbonatos más o menos silicificados o concreciones.

$\mathrm{Su}$ espesor varía lateralmente desde unos $11 \mathrm{~m}$ (Boumia) a unos 70m (Tizi n'Rechou) y en ella se diferencian dos miembros, estando el miembro inferior formado por facies de mayor granulometría (Figs. 3 y 4).

El miembro inferior reposa localmente sobre el complejo basáltico a través de varios niveles calizos silicificados y con hematites. Este miembro es arenoso-conglomerático al SE con mayor abundancia de conglomerados, mientras que hacia el NO predominan las arcillas, se inicia al SE (columnas BK y TW) con conglomerados, poco organizados, con estratificación generalmente muy poco marcada, conglomerados con estratificación cruzada en artesa y con fragmentos angulares a subangulares. Estos fragmentos ocupan más del $85 \%$ del sedimento y son de talla milimétrica a decimétrica, mientras que la matriz, roja, es inmadura y arenosa. 


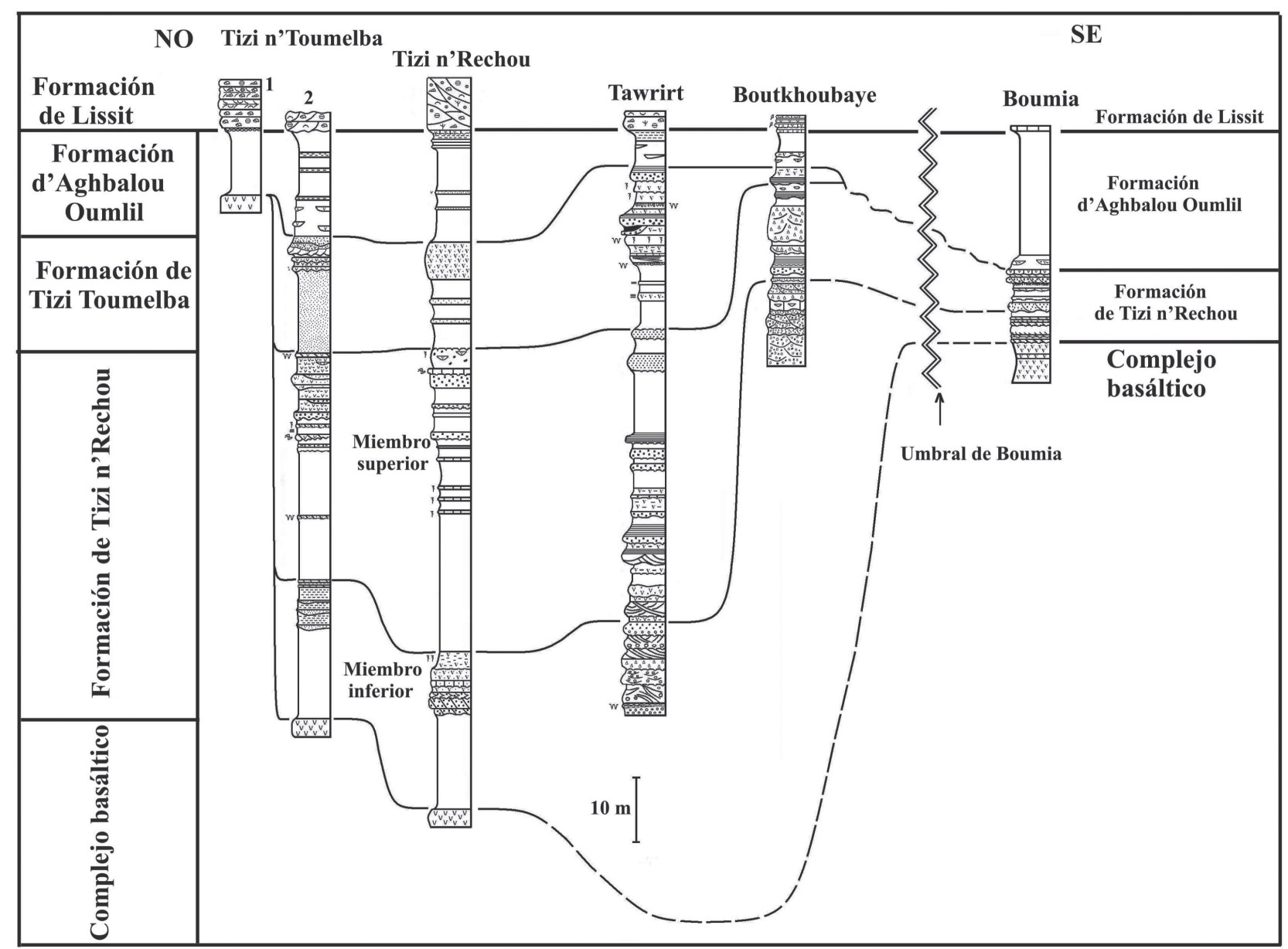

Fig. 3.-Correlación de los depósitos triásico-liásicos de la región de Boumia-Tizi n’Rechou, por encima del complejo basáltico. Las sucesiones terrígenas del Triásico superior- Jurásico basal (STL) están formadas por las formaciones de Tizi n’Rechou, Tizi n'Toumelba y Aghbalou Oumlil (Para la leyenda cf. Fig. 4).

Se organiza en bancos métricos a plurimétricos grano y estratodecrecientes, entre los que se intercalan microconglomerados con fragmentos de basaltos, brechas piroclásticas violáceas con trazas de tubitos de desgasificación y tobas gruesas negruzcas con estratificaciones cruzada muy poco marcadas. También se intercalan areniscas rojas, basaltos y lutitas y concreciones silíceas. Existe también un nivel de calizas nodulosas con concreciones silíceas gris-verdosas y de calizas tobáceas concrecionadas con fragmentos de basalto dolerítico. Esta base queda cubierta por areniscas gruesas con lentejones de brechas y estratificación cruzada plana o cruzada de bajo ángulo. Las areniscas son gruesas hacia la base y tienen fragmentos de basalto dolerítico cuya talla media es de 0,1 a $2 \mathrm{~mm}$, con formas irregulares. Hacia el techo la estratificación pasa a ser laminada.
Estos depósitos cambian lateralmente al NO (columnas Tt1 y TN) a ser lutitas plurimétricas de color rojo ladrillo, cubiertas por una alternancia de lutitas verdosas y de lentejones de arenisca o bien a areniscas gruesas con estratificación cruzada en artesa, organizadas en bancos pluridecimétricos entre los que se intercalan tobas laminadas rojas, tobas finas con geodas y lapilli gris calizas tobáceas, calizas silíceas y dolomías arenosas.

Este miembro corresponde al SE a abanicos aluviales (Figs. 4 y 5) que en la parte superior pasan a ser depósitos formados con un sistema fluvial trenzado, testimonio de un desmantelamiento intenso de los relieves vecinos entonces rejuvenecidos. Durante las avenidas los materiales detríticos gruesos se acumulaban en las zonas subsidentes próximas, mientras que la acumulación al NO de sedimentos de grano fino testimonia la instauración de una lla- 


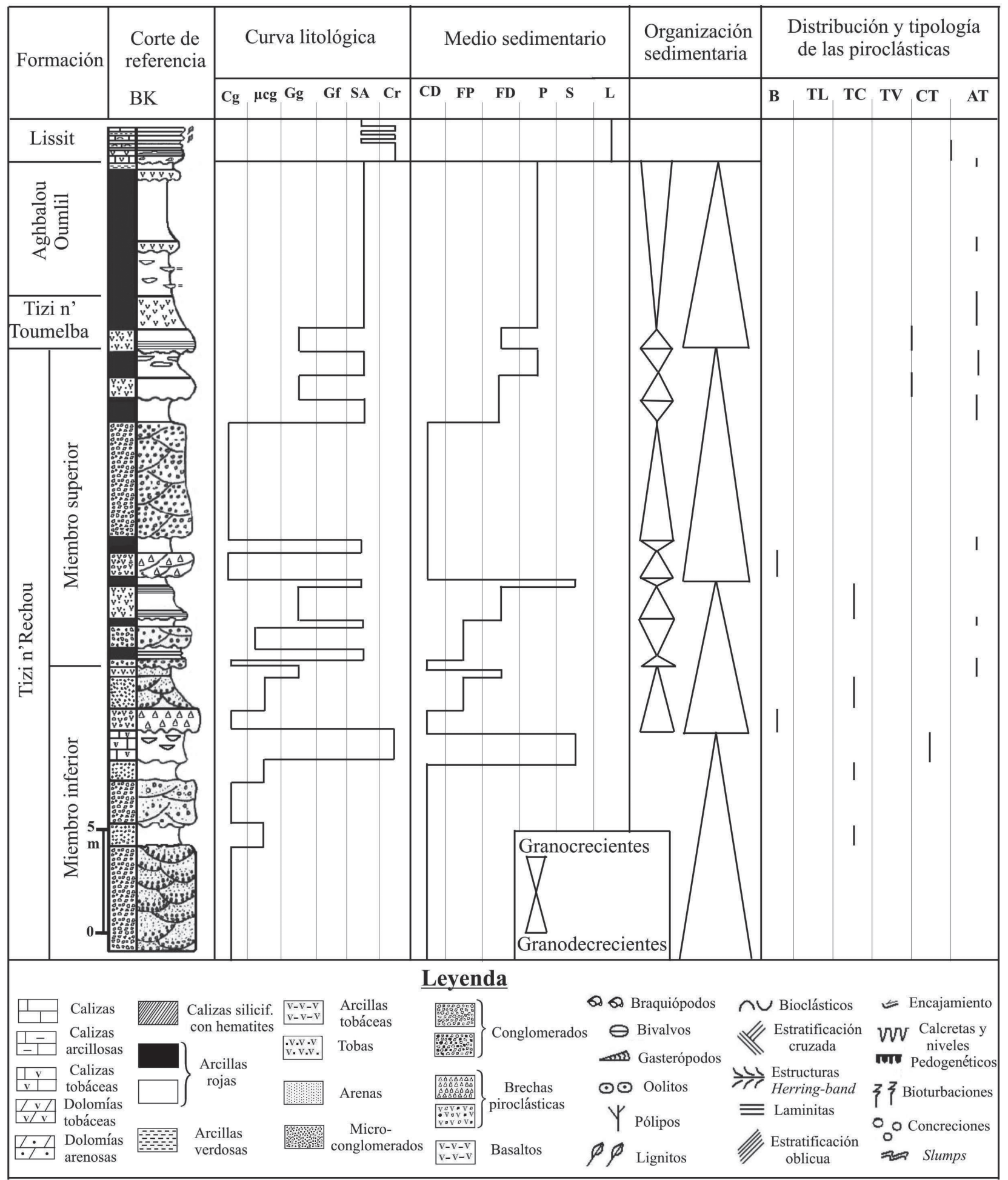

Fig. 4.- -Las STL de la región de Boumia-Tizi n’Rechou: Parámetros sedimentarios y modalidades de la organización de los depósitos. Litologías: Cg: conglomerados, ?cg: microconglomerados, Gg: arenas gruesas, Gf: arenas finas, SA: limos y arcillas, Cr: carbonatos. Medios sedimentarios: CA: abanicos, FP: depósitos fluviales proximales, FD: depósitos fluviales distales, P: llanura de inundación, S y L: depósitos de plataforma de mareas (S: secuencia de tipo Sebkha, L: secuencia de lignitos). Rocas piroclásticas: B: brecha, TL: toba lítica, TC: toba cristalina, TV: toba vítrea, CT: carbonatos tobáceos, AT: lutitas tobáceas. 
nura de inundación alejada del área fuente, llanura en las que produjeron diversas fases pedogenéticas (Fig. 5).

Las variaciones de espesor y los cambios de facies importantes que presentan estos depósitos (Fig. 3), testimonian la reactivación de la actividad tectónica de la región; las facies indican también un clima tropical con alternancia de estaciones húmedas y secas (Millot et al., 1959 y 1977; Bocquier, 1973; Nahon, 1976; Schmitt \& Thiry, 1977; Ben Mlih, 2004; Ben Mlih et al., 2004) y en régimen de rexistasia.

El miembro superior se caracteriza por una alternancia de niveles que van desde las arcillas a los conglomerados, pasando por niveles limosos y arenosos. Los niveles de arcillas son estratocrecientes (pluridecimétricos a plurimétricos), mientras que los niveles gruesos, estratodecrecientes, están limitados a las barras (corte TW. Fig. 3). Estos últimos son a menudo decimétricos a métricos (raramente plurimétricos en el sector del corte BK, Fig. 3) y se biselan lateralmente con bases erosivas.

Se inicia al SE en la base (Fig. 4) con conglomerados de estratificación cruzada planar o en artesa con restos, milimétricos a centimétricos, de basaltos y con una abundante matriz fina. Están cubiertos por bancos pluridecimétricos de areniscas gruesas granodecrecientes, con bases erosivas, y con estratificaciones cruzada en artesa hacia la base y finas y con láminas horizontales hacia el techo. Se intercalan algunos escasos niveles de brechas piroclásticas verdosas, lapilli, tobas gruesas gris-violáceas con pasadas de láminas de toba fina negra y friable. Estos depósitos, más o menos gruesos, se organizan en bancos pluridecimétricos a métricos y entre ellos existen pasadas de lutitas rojo ladrillo, estratocrecientes (de $1 \mathrm{~m}$ a $6 \mathrm{~m}$ de espesor), con pasadas de lutitas tobáceas, localmente laminadas. Los depósitos gruesos, generalmente coronados por los niveles pedogenéticos y calcretas, se disponen en secuencias fluviales trenzados, de proximales a distales (Figs. 4 y 5). El espesor varía (Fig. 3): es reducido en Boutkhoubaye (corte BK) y mayor en Tawrit (corte TW). Esto indica una mayor subsidencia de la cuenca en esa dirección. La variación de las facies y de los espesores testimonian un depósito controlado tectónicamente, con una cierta reactivación de los relieves y también la continuación de la misma dinámica sedimentaria, que se tradujo en el incremento de la erosión de los relieves próximos que se encontraban en reactivación. Al NO, sus depósitos del miembro superior se inician en la base con unas costras de dolomías con sílex, con pasadas de lutitas verdes o rojas, a veces laminadas, costras calizas laminadas, calcretas negras, dolomías tobáceas, calizas muy bioturbadas y arcillas y limos ferruginizados. Estos sedimentos están cubiertos por lutitas rojo-ladrillo con pasadas de tobas negras y de lutitas carbonatadas y laminadas, entre las que se intercalan conglomerados poligénicos con fragmentos centimétricos de basaltos y de carbonatos, microconglomerados con fragmentos centimétricos de calizas, lutitas verdosas y restos de basaltos, areniscas gruesas rojas y tobas con lapilli.

El miembro superior de la formación de Tizi n'Rechou termina con calizas brechificadas con láminas deformadas sobre las que se disponen lutitas rojizas concrecionadas con lentejones de areniscas y culmina en un nivel bioturbado, un paleosuelo, o en unas areniscas con nódulos de sílex con pasadas de lutitas tobáceas concrecionadas o en una calcreta beige. Estos depósitos esencialmente lutíticos atestiguan igualmente la instalación de una llanura de inundación en las que se produjeron diversas fases pedogenéticas (Fig. 5).

El conjunto de estos sedimentos se acumularon en una cuenca intracontinental asimétrica (Figs. 5 y 6) en la que se producía una profundización gradual desde el SE al NO gracias a la existencia de escalones sucesivos, cuya mayor profundidad se situó en Tawrit. Entre esos sedimentos se depositaron rocas piroclásticas (brechas piroclásticas y tobas, con estructuras vitroclásticas), procedentes de emisiones volcánicas explosivas. Son abundantes sobre todo cerca de la base de esta formación, donde hay clastos (epiclastos) más gruesos. Esto ocurre particularmente en el SE, lo que testimonia la proximidad de las emisiones volcánicas (Fig. 4). Por el contrario, al NO, estos depósitos se reducen a algunas finas pasadas de tobas procedentes de la fragmentación de la lava y su expulsión a gran altura (Chalot-Prat \& Legall, 1978), o en algún caso a partir de avalanchas piroclásticas que avanzaron hasta puntos muy lejanos (Fisher \& Schmincke, 1984; Smith, 1986; Schneider, 1990). La abundancia de fragmentos vítreos atestigua el transporte por fluidificación gaseosa del magma (Parsons, 1969), mientras que la de cristales de plagioclasa, en lo más alto del miembro inferior, testimonia la desintegración explosiva de una lava con una cristalización avanzada (Lucas et al., 1976).

La formación de Tizi n'Toumelba (Fig. 3): Bien desarrollada al NO, presenta generalmente sedimentos detríticos finos, con intercalaciones arenosas 


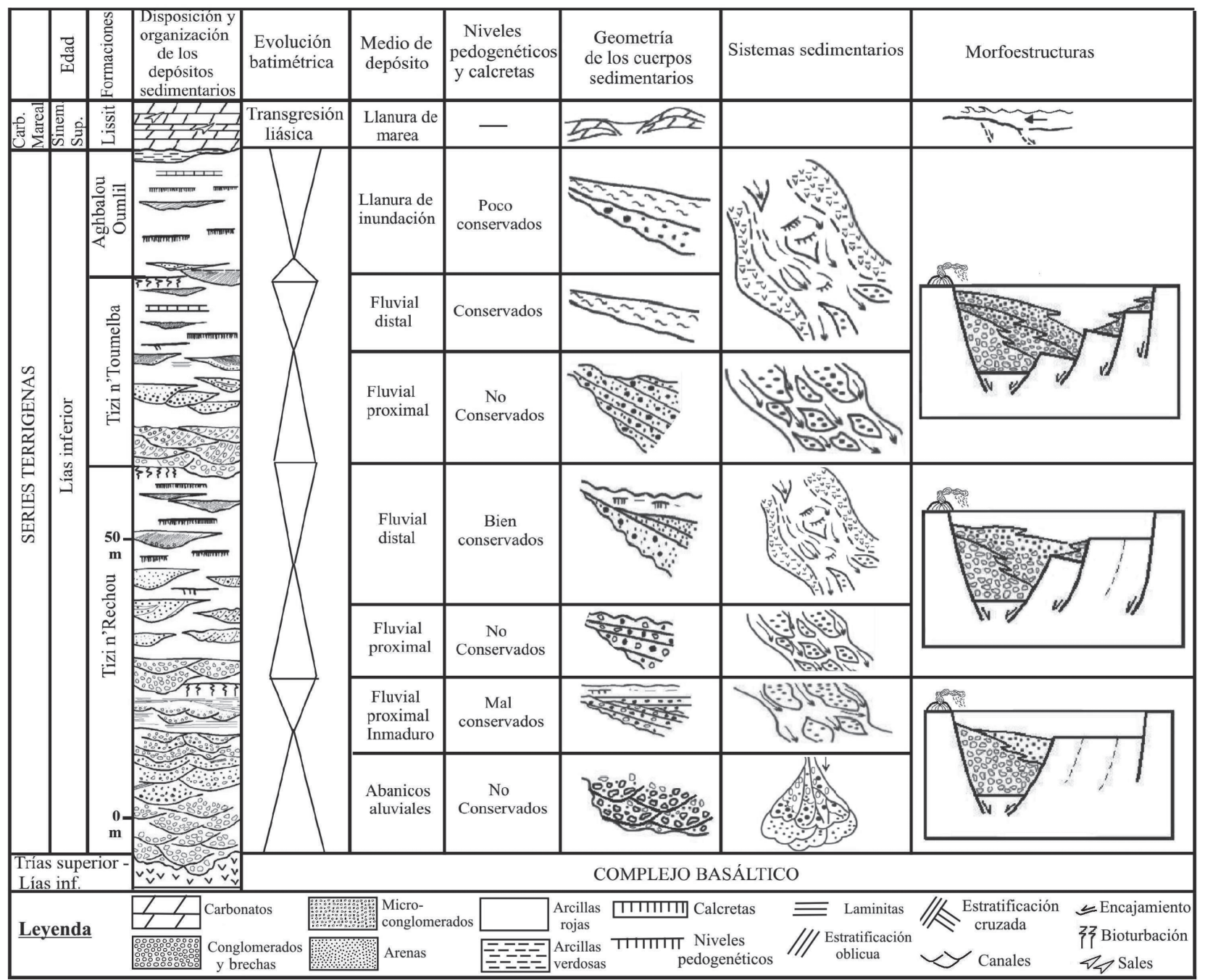

Fig. 5.-Modelo de evolución vertical de las STL durante el Triásico superior-Liásico inferior: sistemas sedimentarios asociados.

NO $\mathbf{T t 1}$ TN TW BK

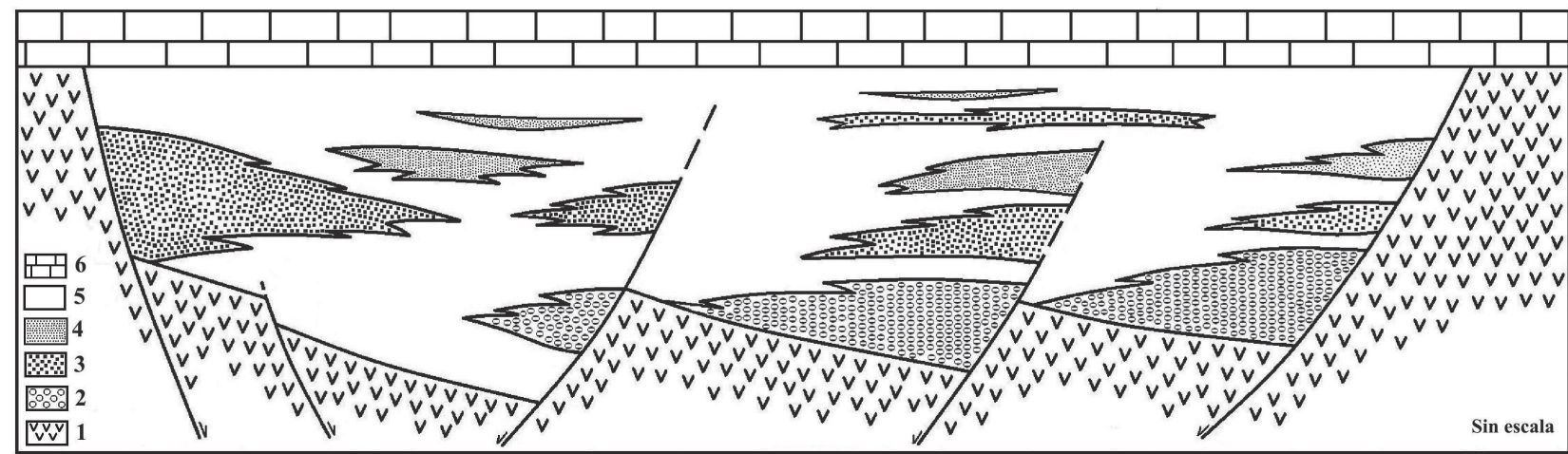

Fig. 6.-Región de Boumia-Tizi n’Rechou: Esquema de la estructuración en bloques basculados. 1- Complejo basáltico, 2- Depósitos de abanicos aluviales, 3- Depósitos fluviales trenzados proximales, 4-Depósitos fluviales trenzados distales, 5-Depósitos arcillolimosos de llanura de inundación, 6-Carbonatos de llanuras de mareas. Su posición correspondería a la línea marcada en la figura 2. 


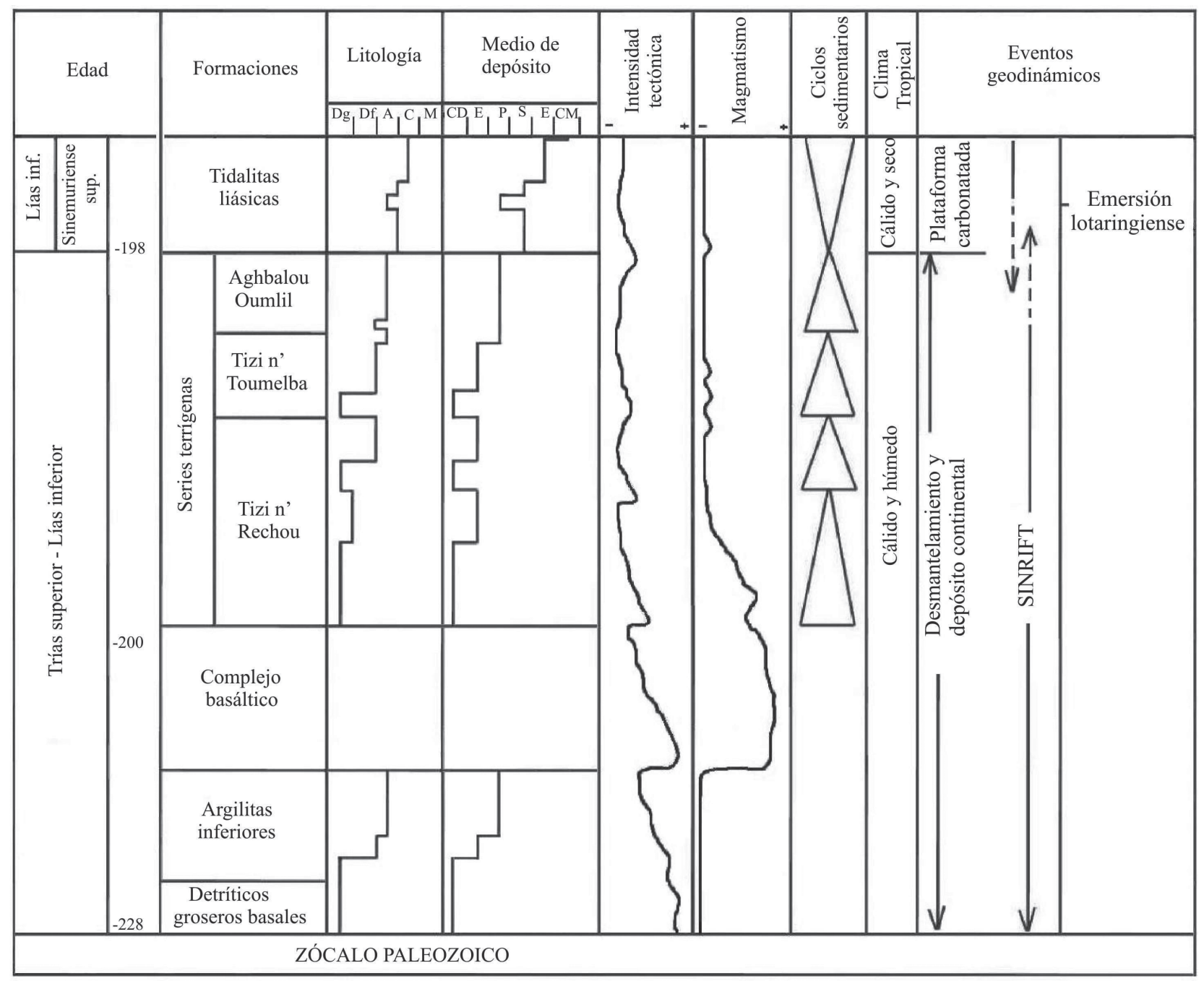

Fig. 7.-Eventos ocurridos durante el depósito de las series triásico-liásicas del Alto Muluya en el borde meridional del Atlas Medio. Litologías- Dg: sedimentos detríticos gruesos, Df: detríticos finos, A: lutitas, C: carbonatos, M: margas. Medios de depósitos: C: abanico, F: depósitos fluviales, P: llanura de inundación, S y L: depósitos de plataforma de mareas (S: secuencia de tipo Sebkha, L: secuencia de lignitos), E: estrán, CM: canal de marea.

dispersas, a las que se asocian cenizas pardo-amarillentas que provienen de explosiones violentas (Poirier et al., 1994) y que pueden permanecer mucho tiempo en suspensión en el aire o en el agua y depositarse a decenas de kilómetros, muy lejos de la fuente volcánica (Fisher \& Schmincke, 1984; Cas \& Wright, 1987).

La formación de Aghbalou Oumlil (Figs. 3, 4 y 5): Presenta lutitas rojo-ladrillo con algunas raras intercalaciones de lutitas consolidadas y tobas, arenas arcillosas y conglomerados poligénicos con fragmentos de basaltos, y de rocas carbonatadas. A techo, en muchos puntos se formaron lutitas verdosas y negruzcas. Esta formación muestra (Fig. 3) una gran extensión regional (Fig. 3) y es testimonio de una notoria homogenización de las facies, propias de una llanura de inundación (Fig. 5 y 7) bajo un clima hidrolizante. La abundancia de depósitos finos indica una importante fase de retrogradación, producida por la maduración del sistema sedimentario, incluida la red fluvial, y la calma tectónica y volcánica que tuvo lugar.

Las lutitas verdosas que terminan la formación atestiguan una inundación marina de la región y la instauración de un medio de tipo sebkha. La incursión marina evolucionó a una transgresión posteriormente durante el depósito de los carbonatos propios de llanuras de mareas (Tidal Flats) del Jurásico 
más bajo. Una débil actividad volcánica se observa aún en la base de los carbonatos donde existen algunas intercalaciones de niveles piroclásticos, representados por carbonatos bioclásticos tobáceos, tobas cristalinas y tufitas.

Las STL están cubiertas por barras carbonatadas (Figs. 3, 5 y 6) del Jurásico inferior (base de los carbonatos ante-Itzer de la Fm de Lissit: Saâdi et al., 1993, 1997, 2003; Saâdi \& Fedan, 1997a y 1999a) con la que muestran un cambio neto (Boumia, Boutkhoubaye, Tawrirt, Tizi n'Toumelba 2) o progresivo (ribera derecha del Oued Kiss y en Tizi n'Toumelba 1).

Estos carbonatos, que se incluyen en la formación de Lissit, son bioclásticos y oolíticos, gris verdoso, presentan estratificación y laminación cruzada y contienen braquiópodos, bivalvos, pólipos aislados, lignito y gasterópodos. Entre estos fósiles, que permiten datar el Lotharingiense medio, se han determinado: Spiriferina praerostrata Flam., Spiriferina sp. var. alpina, Terebratula mediterranea Canav. var. pectita Dub., T. aff. semiarata Dub., Zeilleria cf. arethusa Di Stefano, Rhynchonella moghrabiensis Dub., $R$. cf. moghrabiensis Dub. y Davidsonella sp. (determinaciones de DU DRESNAY, in Saâdi, 1996; Saâdi et al., 2003). Se organizan en bancos métricos a plurimétricos entre las que se intercalan pasadas pluridecimétricas de laminitas calizas rosadas con estructuras de tipo bird's eye, calizas o dolomías tobáceas amarillentas, calizas tableadas y pseudovacuolares o, localmente, dolomías nodulosas verdosas o tobas finas amarillentas (corte de Tizi n'Rechou). Al SE (corte de Boutkhoubaye) pasan a ser una alternancia de lutitas verdosas con lignito y calizas tobáceas amarillentas con lignito y gasterópodos, entre las que se intercalan dos pasadas de tobas amarillentas (Fig. 4).

Sigue a continuación una alternancia de lutitas verdosas y dolomías tobáceas hojosas, amarillentas, con bivalvos y braquiópodos, y dolomías bioclásticas que son cubiertas por calizas y dolomías oolíticas y bioclásticas grisáceas, con braquiópodos, gasterópodos y foraminíferos, además de otros restos fósiles.

Estos carbonatos de mareas se organizan en secuencias de tipo sebkha (Boumia), de lignitos (Boutkhoubaye), y de canales de marea (Tawrirt, Tizi n'Rechou y Tizi n'Toumelba). Esta primera recurrencia carbonatada denota una primera inmersión de la región que se traduce por la instalación de una llanura de mareas tras el ascenso eustático del inicio del Jurásico (Sinemuriense superior: Kaoukaya et al., 2001; Saâdi, 1996; Saâdi et al., 2003). Una débil actividad volcánica perduró en la base de estos carbonatos con algún depósito de niveles piroclásticos representados por tobas y tufitas. Los cambios de facies, las variaciones de espesor y los últimos depósitos volcánicos fueron aun controlados por la reactivación de estructuras previas.

Después, toda la región quedó definitivamente inundada tras el ascenso eustático del Sinemuriense superior, y en ella se instalaron ambientes perimareales, de plataforma marina interna, muy somera y restringida, y de marisma costera (Figs. 5, 6 y 7).

\section{Organización sedimentaria}

Las STL se estructuran como una megasecuencia regional, que comienza con depósitos gruesos que van pasando a otros más finos hacia el techo.

Los depósitos gruesos (miembro inferior de la formación Tizi n'Rechou) se inician con estratos con bases canalizadas, y se organizan en secuencias elementales cuya tendencia global es estrato y granodecreciente, generadas como consecuencia de las avulsiones y el relleno progresivo de canales (Fig. 4). Dichas facies gruesas se interpretan como sedimentos propios de abanicos aluviales que se distribuyen (Figs. 5 y 6) a lo largo de escarpes de fallas formados por una continua actividad tectónica, como suele ser frecuente en sistemas de abanicos aluviales descritos en capas rojas continentales de otras edades (Allen, 1965). Estos depósitos se iniciaban como avalanchas en la base de cada secuencia, siendo transportados desde pequeñas distancias por corrientes torrenciales. Hacia arriba de cada secuencia, estas facies son relevadas por facies depositadas por canales trenzados. Finalmente, a techo de las secuencias se intercalan limos y niveles pedogenéticos que testimonian un disminución momentánea de los depósitos gruesos progradantes.

Los términos superiores (miembro superior de la formación Tizi n'Rechou y formaciones suprayacentes) muestran un enriquecimiento en materiales cada vez más finos (Figs. 3 y 4). La organización sedimentaria de las secuencias elementales es netamente estrato y granodecreciente. Estos depósitos responden al gradiente topográfico existente, formándose depósitos cada vez más distales que evolucionaban lateralmente a partir de un sistema fluvial trenzado con facies canalizadas arenosas (Fig. 5). Los depósitos limo-arcillosos corresponden al desbordamiento e inundación. 
La sedimentación estuvo controlada por repetidos episodios de inundación de una llanura muy extensa. Las discontinuidades del techo de las secuencias, marcadas (Fig. 5) por los horizontes pedogenéticos, los encostramientos carbonatados y las calcretas, corresponden a periodos de desecación; la fase carbonatada se origina a partir de la erosión química previa y su precipitación se produjo en un clima cálido que facilitaba la evaporación.

En cuanto a las rocas piroclásticas, son groseras en la base y testimonian un volcanismo fisural, ligado a las principales fallas de los bordes de la cuenca. Por su parte, las cenizas y las lutitas tobáceas existentes hacia el techo de las STL, corresponderían a fuentes más lejanas y se depositaron por simple decantación, tras haber permanecido en suspensión en el aire o en el agua (Fisher \& Schmincke, 1984; Cas \& Wright, 1987). Las manifestaciones volcánicas implican la permanencia del proceso de rifting durante la sedimentación.

El paso de las STL a los carbonatos de llanuras de mareas (formación de Lissit) de la base del Jurásico supone el comienzo de los depósitos de la plataforma carbonatada liásica, con facies de ambientes litorales. Los cambios de facies y las variaciones importantes de espesores indican las migraciones de los surcos más subsidentes, tras nuevas reestructuraciones morfoestructurales producidas por removilización de las fallas de los bordes de la cuenca (e incluso del interior de las mismas), tal como testimonian las rocas piroclásticas situadas en los primeros niveles carbonatados.

\section{Dinámica sedimentaria}

La organización espacio-temporal de los depósitos triásico-jurásicos de la región estudiada muestra la evolución del paleoumbral del Alto Muluya y de las áreas circundantes, particularmente en el sector situado en el borde SE del Atlas medio, durante el periodo del rifting. Esta cuenca, en la que se producían subsidencias diferenciales, fue el asiento de una sedimentación detrítica y volcanosedimentaria (epiclástica y piroclástica), con facies y arquitecturas diversas, cuya distribución estuvo especialmente ligada a un cuadro morfoestructural complejo. En el registro estratigráfico de estos sedimentos se refleja la actividad de las fallas principales: falla de Aït Oufella (FAO) y falla de Boutkhoubaye (FBK), de los bordes.

La cuenca creada era asimétrica (Figs. 3, 5 y 6): en el sector de Boumia-Tizi n'Rechou, tras la emi- sión del complejo basáltico, las fallas provocaron un progresivo hundimiento, formándose varios escalones que se iniciaron cerca de Tizi n'Rechou, alcanzando allí, en el NO, en las proximidades de la falla del borde meridional del Atlas Medio, las mayores subsidencias en cada fase de hundimiento. Esta extensión se prolongaba hacia el SE.

La imagen general que se obtiene de la evolución del depósito de las STL es la siguiente: a causa de los hundimientos de las fallas se formaron los primeros sectores subsidentes asimétricos al pie de los relieves abruptos que formaban los escarpes de las fallas; allí se recogieron las descargas sedimentarias groseras (Fig. 5). El relleno se producía por avenidas intermitentes con fuerte carga de materiales transportados. Buena parte de los mismos procedían de materiales volcanosedimentarios erosionados, que llegaban transportados por diferentes ríos adaptados a la morfología entonces existente.

Lentamente, comenzaron a formarse pequeñas llanuras aluviales que recibían los depósitos fluviales finos, con episodios pedogenéticos múltiples (Fig. 5), mal conservados. Tanto la actividad tectónica que creaba relieves como las condiciones climáticas (clima tropical con alternancia de estaciones: periodos húmedos responsables de la hidrólisis del aumento de la hidrodinámica fluvial y de las descargas de flujo, y periodos cálidos a áridos, responsables de la formación de costras: Millot et al. (1959); Bocquier (1973); Nahon (1976) y Schmitt \& Thiry (1977), explican esta dinámica que se repite muchas veces (Figs. 5 y 6 ).

Pronto el área de depósito se amplió, formándose una importante red fluvial trenzada que aseguró el transporte y la distribución de los materiales según el gradiente topográfico. El predominio de depósitos limosos en el miembro superior de la formación de Tizi n'Rechou pone de manifiesto la retracción de los sistemas de abanico aluvial que caracterizan al miembro inferior de esta formación y la instauración de una llanura de inundación alejada de las áreas-fuente, sede de una importante decantación. En esta llanura se producían episodios de desecación asociados a fases pedogenéticas (Fig. 6) en un clima cálido y temporalmente árido. Estas mismas condiciones persistieron durante el depósito de la formación de Tizi n'Toumelba. Sin embargo, se encuentra muy reducida y está ausente localmente (BM y Tt2), lo que testimonia la continuación de la movilidad.

Durante el depósito de la formación de Aghbalou Oumlil (Fig. 6), la disminución de la tasa de depósi- 
tos groseros favoreció una retrogradación generalizada de los sistemas clásticos, y una homogenización paleogeográfica que queda expresada en la instauración de grandes llanuras de inundación en toda la región. Las lutitas verdosas superiores evocan un medio reductor y el preludio de una inundación progresiva con la formación de salinas litorales, con sedimentos evaporíticos (sabkhas). A la vez, las emisiones volcánicas quedaron restringidas a algunos niveles, lo que atestigua a la vez una actividad tectónica reducida.

Los carbonatos de mareas de la base del Jurásico (formación de Lissit; Fig. 6) confirman la incursión marina, que pronto llegó a ser más importante, desbordando el conjunto de las cuencas del límite Triásico-Jurásico, y dando lugar a la plataforma carbonatada de las cuencas atlásicas. Estos hechos se ligaron al ascenso eustático global ocurrido en el Hettangiense y Sinemuriense.

\section{Conclusiones}

Durante el paso del Triásico al Jurásico, hubo una notable interacción de procesos tectónicos y sedimentarios en el Alto Muluya y en el borde meridional del Atlas Medio. Se caracterizó (Fig. 7) por un contexto geodinámico extensional relacionado con el rifting del Atlántico central (Manspeizer, 1988; Laville, 1988; Laville \& Piqué, 1991; Piqué \& Laville, 1993, 1996; Laville et al., 2004; Oujidi et al., 1997; Mahmoudi \& Bertrand, 2007; Baudon et al., 2009; Quarbous et al., 2009) cuyos efectos llegaron plenamente al Atlas, reactivándose muchas de las fallas que ya habían afectado al basamento herciniano. Esta removilización fue la que individualizó las cuencas del Alto Muluya, cuyo relleno fue a la vez contemporáneo con los procesos extensionales que, a lo largo del Jurásico, determinarían la apertura del Tethys occidental.

Tras la formación del complejo basáltico, en un cuadro estructural activo, las STL, a pesar de su aparente monotonía, muestran en el detalle una neta heterogeneidad litológica que refleja una actuación compleja de la tectónica. En los bordes de las cuencas, particularmente cerca de las fallas que se mostraban activas, los depósitos que predominaron eran conglomeráticos, depositados en ambientes de abanico aluvial y alimentados en buena parte por materiales volcanoclásticos (epiclásticos y piroclásticos). En sectores más alejados de los bordes, predominaron los sedimentos finos, cuya repartición espacio- temporal estuvo también regida por el clima y la dinámica sedimentaria.

Este relleno, netamente sintectónico (synrift: Laville \& Piqué, 1991; Laville et al., 1995, 2004; Bouatmani et al., 2004; Baudon et al., 2009), se producía en un medio continental con descargas detríticas sucesivas que, desde los abanicos aluviales situados al borde de los macizos montañosos, pasaban a formar sistemas aluviales trenzados con las llanuras de inundación cada vez más extensas. Las importantes variaciones de espesor y los notables cambios de facies atestiguan la reactivación de los relieves, lo que se producía por la continuación del proceso del rifting (Fig. 7). A la vez, el clima bajo el que se producía el relleno era tropical, generalmente con alternancia de estaciones húmedas y secas (Millot et al., 1959; Bocquier, 1973; Nahon, 1976; Schmitt \& Thiry, 1977).

Los sedimentos de la STL se organizaron en tres formaciones: la inferior es la de Tizi n'Rechou marcada por la construcción de abanicos aluviales (miembro inferior) que evolucionaban lateralmente y hacia arriba a un sistema fluvial trenzado. Las facies arenosas y arcillosas rojas atestiguan la instalación de un régimen fluvial maduro cuya orientación general fue controlada por las paleopendientes. Los materiales detríticos se nutrían de la erosión de los nuevos relieves creados, en no pocos casos formados por rocas piroclásticas gruesas propias de un volcanismo explosivo precedente muy importante y que continuó durante la sedimentación con eventos de menor entidad que dieron lugar a diversos horizontes tobáceos que aparecen intercalados entre los sedimentos aluviales. La segunda formación, de Tizi n'Toumelba, presenta el mismo régimen y organización sedimentaria. Sin embargo, su extensión regional estuvo marcada por controles tectonosedimentarios. La tercera, formación de Aghbalou Oumlil, está marcada por la instauración de una llanura de inundación que atestigua una neta homogenización paleogeográfica, produciéndose el relleno de los sectores deprimidos residuales y el solapamiento de los depósitos aluviales de llanura de inundación sobre los paleorelieves de los bordes de la cuenca, ya en parte denudados.

Para entonces, las emisiones piroclásticas, testimonio de un volcanismo explosivo que fue coetáneo con la sedimentación, quedaron restringidas en regiones tectónicamente activas (en el área de Boumia-Tizi n'Rechou, así como en otros bordes del Alto Muluya y del SE del Atlas Medio (Ouarhache, 1993, 2002; Ouarhache et al., 1985, 2000, 2002; 
Saâdi et al., 2004a y b, 2006). Este volcanismo tardío, aún en relación con la tectónica distensiva infra-jurásica, se produjo gracias a nuevos movimientos de las fallas principales. Corresponde a las últimas erupciones volcánicas ligadas al rifting triásico (Triásico superior-Jurasico inferior) en las cuencas atlásicas marroquíes.

En su conjunto, destaca la importancia de las grandes fallas en la creación de relieves y en la configuración de las cuencas, tanto en sus bordes como en los bloques basculados que las afectan en el interior. Este factor tectónico junto con el climático, controlaron el relleno sedimentario durante el Triásico superior y Jurásico inferior en el Alto Muluya.

\section{AGRADECIMIENTOS}

Nuestro enorme agradecimiento a los valiosos comentarios, anotaciones y sugerencias que han realizado los revisores de este trabajo, Martín Algarra y Pérez Lopez, que sin duda, han contribuido enormemente a la mejora del manuscrito.

\section{Referencias}

Allen, J.R.L. (1965). The sedimentation and paleogeography of old Red Sandstone of Anglesey. North WalesProc. Yorks. Geol. Soc., 35: 139-185. doi:10.1144/ pygs.35.2.139

Baudelot, S. \& Charriere, A. (1983). Définition et datation palynologique (Héttangien inférieur) de la Formation de Harira, niveau de décollement sous les Formations dolomitiques du Causse moyen-atlasique (Maroc). Comptes Rendus de l'Académie des Sciences de Paris, 296: 1807-1812.

Baudelot, S.; Charriere, A.; Ouarhache, D. \& Sabaoui, A. (1990). Données Palynologiques

Nouvelles concernant l'Ordovicien et le Trias-Lias du Moyen Atlas (Maroc). Géologie Méditerranéenne, 17: 263-277.

Baudon, C.; Fabuel-Perez, I. \& Redfern, J. (2009). Structural style and evolution of a late Triassic rift basin in the Central High Atlas, Morocco: controls on sediment deposition. Geological Journal, 44: 677-691. doi:10.1002/gj.1195

Ben Mlih, A. (2004). Détritisme synrift continental des bassins permo-triasiques à travers le Haut Atlas de Marrakech. Géodynamique et organisation sédimentaire. Doctorat d'Etat Es-Sciences, Université Mohamed V, Faculté Des Sciences, Rabat, 308 pp.

Ben Mlih, A.; Laâdila, M.; El Youssi, M.; El Kochri A. \& Nacili, M. (2004). Le remplissage synrift au Permien et au Trias du bassin de Tahanaout (Haut Atlas de Marrakech, Maroc). Géodynamique et organisation sédimentaire. Estudios Geológicos, 60: 123-138.

Bouatmani, R.; M'Fedal, A.; El Ouarghioui, A.; Medina, F.; El Mourabit, A. \& Daoudi, L. (2004). Les environnements de dépôts des formations triasiques de la région de Meskala (bassin d'Essaouira, Maroc): apport de l'analyse des diagraphies et des carottes. Bulletin de l'Institut Scientifique de Rabat, Section Sciences de la Terre, 26: 49-67.

Bocquier, G. (1973). Genèse et évolution de deux toposéquences de sols tropicaux du Tchad. Interprétation biogéodynamique. Thèse Science Nataturelle, Université Strasbourg (1971) et Mém. Off. Rech. Sci. Tech. Outre-Mer, Paris, 62, 350 pp.

Cas, R. A. F. \& Wright, J.V. (1987). Volcanic successions. Modern and ancient. Chapman \& Hall, London, 445-467. doi:10.1007/978-94-009-3167-1

Chalot-Prat, F. \& Legall, J. (1978). Pétrographie des ignimbrites et des dépôts volcanoclastiques associés dans le Cambrien de l'Est du Massif armoricain armoricain. Bulletin du Bureau des Recherches Géologiques et Minières, deuxième série, section 1, 3: 187205.

Chalot-Prat, F.; Charriere, A. \& Ouarhache, D. (1986). Découverte d'un volcanisme explosif fini-triasique sur la bordure occidentale du Moyen Atlas (Maroc). Revue de la Faculté des Sciences Techniques de Marrakech, section Sciences de la Terre, 2: 127-141.

Courtinat, B. \& Algouti, A. (1985). Caractérisation probable du Sinémurien prés de Telouat (Haut Atlas, Maroc): datation palynologique. Geobios, 18: 857-864. doi:10.1016/S0016-6995(85)80039-5

El Wartiti, M. (1981). Étude géologique des terrains permo-carboniféres et leur Couverture triasico-miocène dans la zone de Tiddas-Souk-es-Sebt Ait Ikkou (bordure NW du Maroc Central). Thése de 3éme cycle. Université Mohamed V, Rabat, 193 pp.

Et-Touhami, M. (1996). Le Trias évaporitique du bassin de Khémisset (Maroc central): géométrie des corps sédimentaires et environnements de dépôts. In: Le Permien et le Trias du Maroc: état des connaissances. Pumag (Medina, F., ed.), 181-199.

Fiechtner, L.; Friedrichsen, H. \& Hammerschmidt, K. (1992). Geochemistry and geochronology of Early Mesozoic tholeiites from Central Morocco. Geologische Rundschau 81: 45-62. doi:10.1007/BF01764538

Fisher, R. V. \& Schmincke, H.U. (1984). Pyroclastics Rocks. Spinger Verlag, Berlin, Heidelberg, 472 pp. doi:10.1007/978-3-642-74864-6

Ganzeev, A.; Grouzdev, V. \& Mitiaev, A. (1978). Structure géologique de la région de la Haute Moulouya. Mines, géologie et énergie, Rabat, 44: 111-114.

Kaoukaya, A (1987). La plate forme carbonatée liasique de la bordure septentrionale du Maroc central (régions de l'Oued Beht, El Kansera, Agourä̈, El Hajeb). Etude sédimentologique et diagénétique. Docteur de 3éme cycle à l'Université de Pau et des Pays de l'Adour, 207 pp.

Kaoukaya, A.; Laadila, M.; Fedan, B. \& Saadi, Z. (2001). La plate-forme carbonatée liasique de la marge 
méridionale du Haut Atlas centro-oriental, au NE d'Errachidia (Maroc); modèle d'organisation des dépôts margino-littoraux. Bulletin de l'Institut Scientifique, Rabat, section Science de la Terre, 23: 27-38.

Lachkar, G.; Ouarhache, D. \& Charriere, A. (2000). Nouvelles donnés palynologiques sur les formations sédimentaires associées aux basaltes triasiques du Moyen Atlas et de la Haute Moulouya (Maroc). Revue de Micropaléontologie, 43: 281-299. doi:10.1016/S0035-1598(00)90147-8

Laville, E. (1988). A multiple releasing and restraining stepover model for the Jurassic strike-slip basin of the Central Hight Atlas (Morocco). In: Triassic-Jurassic rifting. Continental Breakup and the origin of the Atlantic Ocean and passive margins (Manspeizer, W., ed.), Developments in Geotectonics 22, Elsevier, New York, 499-523.

Laville, E. \& Piqué, A. (1991). La distension crustale atlantique et atlasique au Maroc au début du Mésozoïque: le rejeu des structures hercyniennes. Bulletin de la Société Géologique de France, 162: 1161-1171.

Laville, E.; Charroud, A.; Fedan, B.; Charroud, M. \& Piqué, A. (1995). Inversion négative et rifting atlasique: l'exemple du bassin triasique de Kerrouchen (Maroc). Bulletin de la Société Géologique de France, 166: 364-374. doi:10.1016/j.jafrearsci.2003.12.003

Laville, E.; Piqué, A.; Amrhar, M. \& Charroud, M. (2004). A restatement of the Mesozoic Atlasic Rifting (Morocco). Journal of African Earth Sciences, 38: 145-153.

Lucas, G.; Cros, P. \& Lang, J. (1976). Étude microscopique des roches meubles et Consolidées. Les roches sédimentaires. Doin éditeurs, Paris, 503 pp.

Mahmoudi, A. \& Bertrand, H. (2007). Identification géochimique de la province magmatique de l'Atlantique Central en domaine plissé: exemple du Moyen Atlas marocain. Comptes Rendus Geoscience, 339: 545-552. doi:10.1016/j.crte.2007.07.004

Manspeizer, W. (1988). Triassic-Jurrassic rifting and opennig of the Atlantic: an overview. In: TriassicJurassic rifting. Continental Breakup and the origin of the Atlantic Ocean and passive margins, (Manspeizer, W., ed.), Developments in Geotectonics 22, Elsevier, New York, 41-79.

Manspeizer, W.; Pufeer, J. H. \& Cousminer, H. L. (1978). Separation of Morocco and eastern North America: A Triassic-Liassic stratigraphic record. Geological Society of America Bulletin, 89: 901-920. doi:10.1130/0016-7606(1978)89<901:SOMA$\mathrm{EN}>2.0 . \mathrm{CO} ; 2$

Marzoli, A.; Bertrand, H.; Knight, K. B.; Cirilli, S.; Buratti, N.; Vérati, C.; Nomade, S.; Renne, P.R.; Youbi, N.; Martini, R.; Allenbach, K.; Neuwerth, R.; Rapaille, C.; Zaninetti, L. \& Bellieni, G. (2004). Synchrony of the Central Atlantic magmatig province and the Triassic-Jurassic boundary climatic and biotic crisis, Geology, 32: 973-976. doi:10.1130/G20652.1

Michard, A. (1976). Eléments de géologie marocaine. Notes et Mémoires, Service Géologique du Maroc, 256, $420 \mathrm{pp}$.
Millot, G.; Radier, H.; Muller-Feuga, R.; Defossez, M. \& Wey, R. (1959). Sur la géochimie de la silice et les silicifications sahariennes. Bulletin Service Carte géologique, Alsace Lorraine, 12: 236-244.

Millot, G.; Nahon, D.; Paquet, H.; Ruellan, A. \& Tardy, Y. (1977). L'épigénie calcaire des roches silicatées dans les encroûtements carbonatés en pays sub-aride Anti-Atlas, Maroc. Science Geologie Bulletin, Strasbourg, 30: 129-152.

Nahon, D. (1976). Cuirasses ferrugineuses et encroûtements calcaires au Sénégal occidental et en Mauritanie. Systémes évolutifs: géochimie, structures, relais et coexistence. Thése, Science Naturelle, Université AixMarseille, 232 pp.

Ouarhache, D. (1993). La série volcano-sédimentaire de l'Oued Kiss: Moyen Atlas plissé (Maroc). 14 th regional Meeting of sedimentology, I.A.S., Marrakech, April 27-29, 250.

Ouarhache, D. (2002). Sédimentation et volcanismes (effusif et explosif) associés au Rifting triasique et infraliasique dans le Moyen Atlas Sud-occidental et la Haut Moulouya (Maroc). Doctorat d'Etat Es-Sciences, Université Mohamed V, Faculté Des Sciences, Rabat, 284 pp.

Ouarhache, D.; Chalot-Prat, F. \& Charrière, A. (1985). Découverte d'un volcanisme explosif fini-triasique sur la bordure nord-occidentale du Moyen Atlas (Maroc). PICG-UNESCO 183, Marrakech, résumé, 27.

Ouarhache, D.; Charrière, A.; Chalot-Prat, F. \& ElWartiti, M. (2000). Sédimentation détritique continentale synchrone d'un volcanisme explosif dans le Trias terminal à Infra-Lias du domaine atlasique (Haute Moulouya, Maroc). Journal of African Earth Sciences, 31: 555-570. doi:10.1016/S08995362(00)80007-X

Ouarhache, D.; Charrière, A.; Chalot-Prat, F. \& ElWartiti, M. (2002). Relations tectoniques, sédimentation et volcanisme à l'issue du Rinfting triasique en limite du Moyen Atlas et de la Haute Moulouya (Maroc). 19éme Colloque de Géologie Africain, El Jadida, 150-151.

Ouarhache, D; Charrière, A.; Chalot-Prat, F. \& El-Wartiti, M. (2004). Fonctions polyphasées, du Rinfting du Trias-Lias inférieur dans le Moyen Atlas et de la Haute Moulouya (Maroc). 4éme Réunion du Groupe marocain du Permien et du Trias (GMPT-4), Fès, Maroc, 31-32.

Oujidi, M. (1996). Evolution tectono-sédimentaire des Monts d'Oujda (Maroc central) aux tours du Trias et du Lias basal. In: Le Permien et le Trias du Maroc: Etats de connaissances (Medina, F., ed.), Pumg, 201212.

Oujidi, M.; Courel, L.; Benaouiss, N.; El-Mostaine, M.; El-Youssi, M.; Et-Touhami, M.; Jalil, N.; Ouarhache, D.; Sabaoui, A. \& Tourani, A. (1997). La marge PeriTéthysienne marocaine pendant le Trias, Résumé p 34. In: Les Marges téthysiennes d'Afrique du nord. Séance Spécialisée de la Société Géologique de France, du Groupe Français du Crétacé et de la Société Géologique d'Algérie, Paris, 34-35. 
Oujidi, M.; Courel, L.; Benaouiss, N.; El-Mostaine, M.; El-Youssi, M.; Et-Touhami, M.; Ouarhache, D.; Sabaoui, A. \& Tourani, A. (2000). Triassic series of Morocco, stratigraphy, paleogeography and structuring of the Southwestern Per-Tethyan platform. An overview. In: Peri-Tethys Mémoir 5: New Data on Peri-Tethyan Sedimentary Basins (Crasquin-Soleau, S. \& Barrier, E., eds.). Mémoires Muséum Nacional d'Histoire Naturelle, Paris, 182: 23-37.

Parsons, W. H. (1969). Criteria for the recognition of volcanic Breccias: Review in «Igneous and Metamorphic Geology ». Geological Society of America Bulletin, 115: 263-304.

Piqué, A. \& Laville, E. (1993). Les séries triasiques du Maroc, marqueurs du rifting atlantique. Comptes Rendus de l'Académie des Sciences de Paris, 317, Série II: 1215-1220.

Piqué, A. \& Laville, E. (1996). The Central Atlantic rifting: reactivation of Paleozoic structures. Journal of Geodynamics 21: 235-255. doi:10.1016/02643707(95)00022-4

Poirier, J.P.; Besson, P. \& Boudon, G. (1994). Des cendres d'origine phréato-Magmatique dans les dépôts de la crise de 1976-77 de la Soufrière de Guadeloupe. Comptes rendus de l'Académie des Sciences de Paris, 318, série II: 483-486.

Quarbous, A.; Horpffner, Ch. \& Medina, F. (2009). Mise en évidence d'un niveau à méga-slumps au sommet de la série triasique du Bassin de Tizi n'Test (Haut Atlas, Maroc). Bulletin de l'Institut scientifique de Rabat, Section Sciences de la Terre, 31: 35-39.

Rakus, M. (1986). Contribution à la connaissance du Trias moyen-supérieur et du Lias inférieur dans le Maroc oriental. PICG-UNESCO ${ }^{\circ} 183$, Marrakech, 1985, Revue de la Facuté des Sciences, Marrakech, Section Science de la Terre, numero special, 2: 534535.

Saâdi, Z. (1996). Evolution géodynamique triasico-jurassique de la Haute Moulouya et du Moyen Atlas méridional. Place dans l'évolution méso-cénozö̈que du domaine des chaînes atlasiques (Maroc). Thèse de 3ème cycle, Université Mohamed V, Faculté des Sciences de Rabat, 485 pp.

Saâdi, Z. \& Fedan, B. (1997a). Évolution méso-cénozoïque comparée du Moyen Atlas méridional et de la Haute Moulouya, 17ème Colloque des Bassins sédimentaires marocains, Kènitra, 60.

Saâdi, Z. \& Fedan, B. (1997b). Les Bassins triasico-liasiques de type Rift de la Haute Moulouya et du Moyen Atlas méridional. Genèse et Evolution. Première Réunion du Groupe Marocain du Permien et du Trias, Oujda, 50-51.

Saâdi, Z.; Fedan, B. \& Laadila, M. (1999a). Les tidalites liasiques du Moyen Atlas méridional, de Boumia-Aghbalou n'Serdane et de Taddammout-Mibladène. Marqueurs de l'évolution du paléoseuil de la Haute Moulouya. ler Colloque sur le Jurassique marocain, Rabat, 118-119.

Saâdi, Z. \& Fedan, B. (1999b). Les Dépôts triasico-liasiques de la Haute Moulouya et du Moyen Atlas: Typo- logie, Caractérisation et Signification Géodynamique. Deuxième Réunion du Groupe Marocain du Permien et du Trias, Marrakech, 40-41.

Saâdi, Z.; Fedan, B.; Charroud, M. \& Souhel, A. (1993). L'évolution jurassique du Paléoseuil de la Haute Moulouya (Maroc): un exemple d'enregistrement sédimentaire de la tectonique et des variations eustatiques. Congrès international 14th Regional Meeting of Sedimentology (IAS). Marrakech, 291-292.

Saâdi, Z.; Fedan, B.; Laadila, M. \& Lakhbiza, F. (1997). Modalités de l'installation de La plate-forme carbonatée liasique au Maroc. Les Marges téthysiennes d'Afrique du nord. Séance Spécialisée de la Société Géologique de France du Groupe Français du Crétacé et de la Société Géologique d'Algérie, Paris, 35.

Saâdi, Z.; Fedan, B.; Laadila, M. \& Kaoukaya, A. (2003). Les tidalites liasiques de la Haute Moulouya et du Moyen Atlas méridional (Maroc): dynamique sédimentaire et contexte paléogéographique. Bulletin de l'Institut Scientifique, Rabat, section Sciences de la Terre, 25: 55-71.

Saâdi, Z.; Fedan, B.; Laadila, M. \& Zahour, Gh. (2004a). «La série argilo-saliféres supérieures » triasico-liasiques de la Haute Moulouya et du Moyen Atlas méridional: éléments de caractérisation sédimentologique et impacts du climat. Quatrième Réunion du Groupe marocain du Permien et du Trias, Fès, 40.

Saâdi, Z.; Fedan, B. \& Laâdila, M. (2004b). Évolution triasico-liasique de la Haute Moulouya et du Moyen Atlas méridional: héritage structural et modalité tectono-sédimentaire. Quatrième Réunion du Groupe marocain du Permien et du Trias. Fès, 41.

Saâdi, Z.; Fedan, B. \& Laâdila, M. (2006). Les dépôts triasico-liasiques de la Boutonnière de Zeïda-Aouli (Haute Moulouya, Maroc): Typologie, organisation et cadre géodynamique. Cinquième Réunion du Groupe Marocain du Permien et du Trias, El Jadida, Maroc: 71-72.

Sabaoui, A. (1987). Structure et évolution alpine du Moyen Atlas septentrional sur la transversale Tleta des Zerarda. Meghraoua (SW de Taza, Maroc). Thèse de 3éme cycle, Université Paul Sabatier, Toulouse III, $162 \mathrm{pp}$.

Salvan, H. M. (1984). Les formations évaporitiques du Trias marocain. Problèmes stratigraphiques, paléogéographiques et paléoclimatologiques. Quelques réflexions. Revue Géologie Dynamique Géographie Physique, 25: 187-203.

Sebai, A.; Feraud, G.; Bertrand, H. \& Hanes, J. (1991). 40Ar/39Ar dating and geochemistry of tholeiitic magmation related to the early opening of the Central Atlantic Rift. Earth and Planetary Science Letters, 104: 455-472. doi:10.1016/0012-821X(91) 90222-4

Schneider, J. L. (1990). Enregistrement de la dynamique varisque dans les bassins volcano-sédimentaires dévono-dinantiens: exemple des Vosges du Sud (zone Moldanubienne). Thèse, Université de Strasbourg, $222 \mathrm{pp}$. 
Schmitt, J. M. \& Thiry, M. (1977). Minéralisation en Plomb par évolution pédogénetique d'une série arkosique du Trias (Zeïda, Haute Moulouya, Maroc). Bulletin du Bureau de Recherches Géologiques Marocain (deuxième série). Section II, 2: 113-133.

Smith, G. A. (1986). Coarse-grained non marine volcaniclastic sediment: Terminology and depositional process. Geological Society of America Bulletin, 97: 110. doi:10.1130/0016-7606(1986)97<1:CNVS$\mathrm{TA}>2.0 . \mathrm{CO} ; 2$
Verati, Ch.; Bertrand, H. \& Feraud, G. (2005). The farthest record of the central Atlantic Magmatic Province into west africa craton: Precise ${ }^{40} \mathrm{Ar} /{ }^{39} \mathrm{Ar}$ dating and geochemistry of Taoudenni basin intrusives (northern Mali). Earth and Planetary Science Letters 235: 391407. doi:10.1016/j.epsl.2005.04.012

Recibido el 7 de julio de 2010 Aceptado el 21 de marzo 2011 Publicado online el 21 de octubre de 2011 